CHRONIC OBSTRUCTIVE PULMONARY DISEASE

\title{
Patterns of dynamic hyperinflation during exercise and recovery in patients with severe chronic obstructive pulmonary disease
}

\author{
I Vogiatzis, O Georgiadou, S Golemati, A Aliverti, E Kosmas, E Kastanakis, \\ N Geladas, A Koutsoukou, S Nanas, S Zakynthinos, Ch Roussos
}

Thorax 2005;60:723-729. doi: 10.1136/thx.2004.039115

See end of article for authors' affiliations

Correspondence to: Dr I Vogiatzis, National and Kapodistrian University of Athens Medical School, Thorax Foundation, Centre for Applied Biomedical Research and Education, 10675 Athens, Greece; gianvog@phed.voa.gr

Received

14 December 2004

Accepted 2 June 2005

Published Online First

17 June 2005
Background: Not all patients with severe chronic obstructive pulmonary disease (COPD) progressively hyperinflate during symptom limited exercise. The pattern of change in chest wall volumes (Vcw) was investigated in patients with severe COPD who progressively hyperinflate during exercise and those who do not.

Methods: Twenty patients with forced expiratory volume in 1 second $\left(\mathrm{FEV}_{1}\right) 35(2) \%$ predicted were studied during a ramp incremental cycling test to the limit of tolerance (Wpeak). Changes in Vcw at the end of expiration ( $E E V \mathrm{Cw}$ ), end of inspiration (EIV Cw), and at total lung capacity ( $T L C V \mathrm{CW}$ ) were computed by optoelectronic plethysmography (OEP) during exercise and recovery.

Results: Two significantly different patterns of change in $\mathrm{EEV} c w$ were observed during exercise. Twelve patients had a progressive significant increase in EEVcw during exercise (early hyperinflators, EH) amounting to $750(90) \mathrm{ml}$ at Wpeak. In contrast, in all eight remaining patients EEV cw remained unchanged up to $66 \%$ Wpeak but increased significantly by $210(80) \mathrm{ml}$ at Wpeak (late hyperinflators, $\mathrm{LH})$. Although at the limit of tolerance the increase in $\mathrm{EEV} \mathrm{cw}$ was significantly greater in $\mathrm{EH}$, both groups reached similar Wpeak and breathed with a tidal EIV cw that closely approached TLCV $\mathrm{cw}$ (EIV $\mathrm{cw} / \mathrm{TLCV}$ cw $93(1) \%$ and $93(3) \%$, respectively). EEV cw was increased by 254 (130) $\mathrm{ml}$ above baseline 3 minutes after exercise only in $\mathrm{EH}$.

Conclusions: Patients with severe COPD exhibit two patterns during exercise: early and late hyperinflation. In those who hyperinflate early, it may take several minutes before the hyperinflation is fully reversed after termination of exercise.
$\mathrm{P}$ rogressive dynamic hyperinflation leads to intolerable sensations of breathlessness that make an important contribution to the limitation of symptom limited exercise in most patients with chronic obstructive pulmonary disease (COPD). ${ }^{1}$ In these patients changes in end expiratory lung volume (EELV) constitute an important outcome in assessing the effects of therapeutic interventions on the development of dynamic hyperinflation during exercise. ${ }^{2-4}$ The assessment of dynamic changes in EELV is routinely carried out by serial inspiratory capacity (IC) manoeuvres ${ }^{56}$ assuming that, in patients with COPD, total lung capacity (TLC) does not change appreciably during exercise. ${ }^{78}$

On the other hand, there are a significant number of patients with COPD who do not hyperinflate progressively during exercise ${ }^{6-12}$ but still claim dyspnoea as the main cause of exercise limitation. ${ }^{6}$ The results reported for this category of COPD patients are, however, discrepant as EELV has either been reported to remain constant with increasing intensity ${ }^{12}$ or actually to fall, ${ }^{11}$ as is commonly seen in healthy subjects. ${ }^{13}$ Accordingly, exercise limitation in these patients is not associated with end expiratory dynamic hyperinflation. This implies that simply tracking changes in EELV during exercise is not informative of all the factors that intensify dyspnoea and reduce exercise capacity in these patients. In COPD patients there is also variability in the response of the end inspiratory lung volume (EILV) to exercise: most studies report a progressive increase in EILV $^{15612}$ although Aliverti et $a l^{11}$ found that some patients exhibit a stable EILV. Assessment of all dynamically modified operational lung volumes during exercise is therefore important for understanding which factors contribute to exercise limitation. This study was undertaken primarily to identify possible differences in the pattern of response in operational volumes during exercise in patients with severe COPD.

Optoelectronic plethysmography (OEP) is a technique capable of accurately measuring breath by breath changes in the volumes of the entire chest wall (Vcw) and its rib cage and abdominal chest wall compartments. ${ }^{13-15}$ In addition, OEP can measure breath by breath variations in end inspiratory and end expiratory Vcw and volume variations of the different chest wall compartments. These measures are crucial for understanding the different ventilatory strategies adopted during exercise between different patients. OEP can also track any changes in VCW at TLC (TLCVCW) if maximal inspirations are repeatedly made during exercise. Thus, one can determine if tidal volume is restricted when end inspiratory volume is at or near TLC. As the literature is lacking research investigating changes in operational lung volumes following the cessation of exhaustive exercise in

Abbreviations: $\mathrm{EEV}_{\mathrm{cw}}$, end expiratory chest wall volume; $\mathrm{EFL}$, expiratory flow limitation; ElVcw, end inspiratory chest wall volume; EH, early hyperinflator; $\mathrm{fb}$, breathing frequency; $F E V_{1}$, forced expiratory volume in 1 second; FVC, forced vital capacity; FRC, functional residual capacity; IC, inspiratory capacity; IRVcw, inspiratory reserve chest wall volume; $L H$, late hyperinflator; OEP, optoelectronic plethysmography; RER, respiratory exchange ratio; RV, residual volume; TLCO, carbon monoxide lung transfer factor; TLC, total lung capacity; TLCV CW, chest wall volume at total lung capacity; $\mathrm{V}_{\mathrm{cw}}$, chest wall volume; $\dot{\mathrm{V}}_{\mathrm{E}}$, minute ventilation; $\dot{V}_{2}$, oxygen uptake; $V_{T}$, tidal volume; Wpeak, peak workload. 
patients with COPD, we also investigated the pattern of change in Vcw during recovery from exercise since this could be an important issue for patients when dealing with activities of daily living.

\section{METHODS \\ Subjects}

The study patients included 15 men and 5 women with stable COPD who satisfied the following criteria: (1) post-bronchodilator forced expiratory volume in 1 second $\left(\mathrm{FEV}_{1}\right)<50 \%$ predicted and ratio of $\mathrm{FEV}_{1}$ to forced vital capacity (FVC) $<65 \%$ without significant reversibility $(<12 \%$ change of the initial $\mathrm{FEV}_{1}$ value); (2) optimised medical treatment; and (3) no clinical evidence of exercise limiting cardiovascular or neuromuscular diseases. Patients signed an informed consent form and the protocol was approved by the University Ethics Committee.

\section{Pulmonary function assessment}

Spirometric tests and measurement of lung transfer factor for carbon monoxide (TLCO) were performed by a spirometer (Masterlab; Jaeger, Wurzburg, Germany) while subdivisions of lung volumes were measured by body plethysmography (Medgraphic Autolink 1085D, Medical Graphics, St Paul, MN, USA) according to ATS standards. ${ }^{16}$

\section{Exercise protocol}

The following incremental protocol was performed on an electromagnetically braked cycle ergometer (Ergoline 800; Sensor Medics, Anaheim, CA, USA): after 3 minutes of measurements during quiet breathing, followed by 3 minutes of unloaded pedalling, the work rate was increased every minute (increments of 5 or $10 \mathrm{~W}$ ) to the limit of tolerance (Wpeak) while patients maintained a pedalling frequency of $60 \mathrm{rpm}$. The following gas exchange and ventilatory variables were recorded breath by breath (Vmax 229, Sensor Medics): oxygen uptake $\left(\dot{\mathrm{V}}_{2}\right)$, carbon dioxide output $\left(\dot{\mathrm{V}}_{\mathrm{CO}_{2}}\right)$, respiratory exchange ratio (RER), minute ventilation $(\dot{\mathrm{V}} \mathrm{E})$, tidal volume $(\mathrm{VT})$, and breathing frequency $(\mathrm{fb})$. Cardiac frequency (fc) and percentage oxygen saturation $\left(\mathrm{Spo}_{2} \%\right)$ were determined using the $\mathrm{R}-\mathrm{R}$ interval from a 12-lead on line electrocardiogram (Marquette Max; Marquette Hellige GmbH, Germany) and a pulse oximeter (Nonin 8600; Nonin Medical, USA), respectively. The modified Borg scale ${ }^{17}$ was used to rate the magnitude of dyspnoea and leg discomfort every 2 minutes throughout exercise.

\section{Operational lung and chest wall volume measurements}

At baseline, during unloaded cycling and incremental exercise, patients performed IC manoeuvres at quiet breathing, every 2 minutes during exercise, and in recovery. Patients were instructed after 3-4 regular tidal breaths to make maximal IC efforts from EELV to TLC according to previously described methods. ${ }^{1}$ Simultaneously, chest wall kinematics were measured by OEP as previously described. ${ }^{13-15}$ In brief, the movement of 89 retro-reflective markers placed front and back over the chest wall from clavicles to pubis was recorded. Each marker was tracked by six video cameras (Smart System BTS, Milan, Italy), three in front of the subject and three behind. Subjects grasped handles positioned at the mid sternum level which lifted the arms away from the rib cage so that lateral markers could be visualised. Dedicated software reconstructs the three-dimensional coordinates of the markers in real time by stereophotogrametry and calculates total and compartmental chest wall volume and volume variations using Gauss's theorem. As in the study by Aliverti and coworkers, ${ }^{11}$ the chest wall was modelled as being composed of two compartments - the rib cage and the abdomen. Vcw was the sum of the rib cage volume (Vrc) and abdominal volume (Vab). ${ }^{11}$ Vcw data are reported during quiet breathing, unloaded cycling (0 Watts), at 33\%,66\% and $100 \%$ of peak exercise workload (Wpeak), and 1 minute (Rl) and 3 minutes (R2) into the recovery.

\section{Comparison of OEP with spirometric data}

$\mathrm{VT}$ measured by the OEP ( $\mathrm{VT}_{\mathrm{OEP}}$ ) was calculated as the difference between end inspiratory and end expiratory Vcw (EIVCw - EEVCW). As in a previous study ${ }^{15}$ in which we assessed the ability of the OEP to measure changes in lung volumes during exercise, we compared $\mathrm{VT}_{\mathrm{TEEP}}$ with $\mathrm{VT}$ obtained spirometrically ( $\mathrm{VT}_{\mathrm{SP}}$ ) over periods of 20 seconds throughout all stages. IC was calculated by the OEP ( $\mathrm{IC}_{\mathrm{OEP}}$ ) as the difference between TLCVCw and the EEVcw; the latter value was derived by averaging the EEVCW over a period of 20 seconds before the IC effort (fig 1). $\mathrm{IC}_{\mathrm{OEP}}$ and EEVCW values recorded at quiet breathing, during exercise and recovery were compared with IC measured by the spirometer ( $\left.\mathrm{IC}_{\mathrm{SP}}\right)$ according to previously described methods. ${ }^{1}$

\section{Statistical analysis}

Data are presented as mean (SE) values. Linear regression analysis was performed using the least squares method. Two way analysis of variance (ANOVA) with repeated measures was used to identify statistically significant differences in chest wall volumes across different time points between groups. Within groups one way ANOVA with repeated measures was performed to examine statistical differences, followed by paired $t$ tests when necessary. For all analyses a statistical significance of 0.05 was used, with appropriate Bonferroni corrections for multiple comparisons.

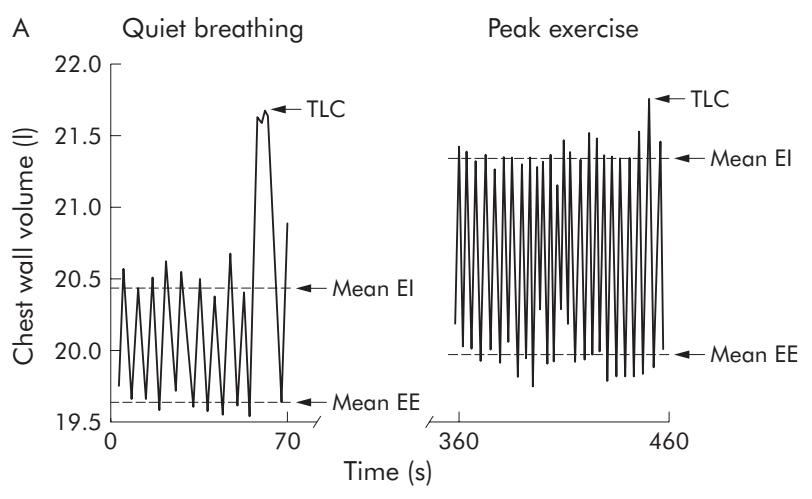

B

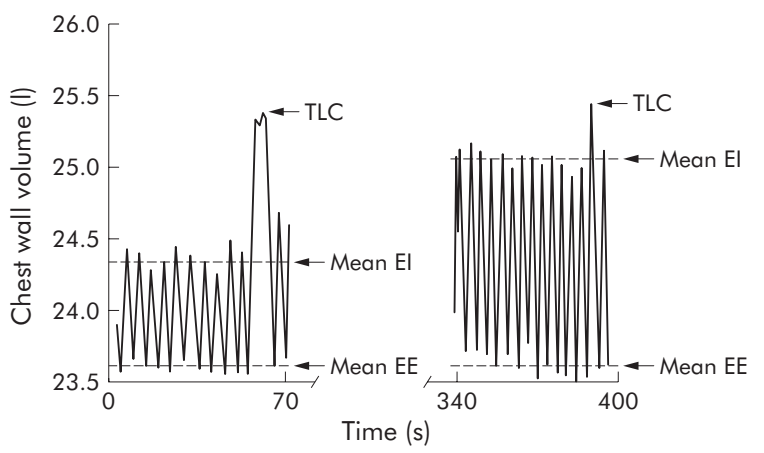

Figure 1 Typical experimental tracings of absolute chest wall volume measurements obtained from (A) an early hyperinflator and (B) a late hyperinflator patient during quiet breathing and peak exercise. $A$ gradual shift in volumes during exercise occurred because of an increase in mean end inspiratory (EI) and mean end expiratory (EE) chest wall volumes indicated by the dashed line. Chest wall volumes at total lung capacity (TLC) are indicated by an arrow. 
Table 1 Mean (SE) demographic and post-bronchodilator data of the study population and subgroups

\begin{tabular}{llll}
\hline & $\begin{array}{l}\text { COPD } \\
(\mathbf{n}=20)\end{array}$ & $\begin{array}{l}\text { Early hyperinflators } \\
(\mathbf{n}=12)\end{array}$ & $\begin{array}{l}\text { Late hyperinflators } \\
(\mathbf{n}=8)\end{array}$ \\
\hline Age (years) & $62(2)$ & $61(3)$ & $64(2)$ \\
Height (cm) & $167(2)$ & $168(3)$ & $165(3)$ \\
Weight (kg) & $66(2)$ & $65(3)$ & $67(4)$ \\
BMI (kg/m $\left./ \mathrm{m}^{2}\right)$ & $23.7(0.7)$ & $22.9(1.0)$ & $24.7(0.7)$ \\
FEV $1($ (I) & $0.94(0.07)$ & $0.93(0.10)$ & $0.94(0.09)$ \\
FEV (\% pred) & $35(2)$ & $33(4)$ & $37(7)$ \\
FVC (I) & $2.70(0.13)$ & $2.65(0.19)$ & $2.76(0.15)$ \\
FVC (\% pred) & $79(4)$ & $76(5)$ & $84(5)$ \\
FEV $/$ FVC (\%) & $35(2)$ & $35(3)$ & $35(3)$ \\
TLCO (\% pred) & $43(6)$ & $37(4)$ & $49(13)$ \\
TLC (\% pred) & $120(22)$ & $123(13)$ & $119(16)$ \\
FRC (\% pred) & $156(14)$ & $157(11)$ & $149(10)$ \\
RV (\% pred) & $216(10)$ & $220(12)$ & $212(9)$ \\
IC (I) & $2.07(0.08)$ & $2.03(0.12)$ & $2.15(0.08)$ \\
IC (\% pred) & $70(4)$ & $69(7)$ & $71(5)$ \\
\hline
\end{tabular}

$\mathrm{FEV}_{1}$, forced expiratory volume in 1 second; FVC, forced vital capacity; TLCO, carbon monoxide lung transfer factor; TLC, total lung capacity; FRC, functional residual capacity; RV, residual volume; IC, inspiratory capacity.

\section{RESULTS}

\section{Patient characteristics}

Patients were characterised by severe airway obstruction and a reduction in TLCO with increased TLC, functional residual capacity (FRC) and residual volume (RV) (table 1). Exercise capacity was severely compromised (table 2 ).

\section{Comparison of OEP with spirometric data}

The relationship between $\mathrm{VT}_{\mathrm{OEP}}$ and $\mathrm{VT}_{\mathrm{SP}}$ calculated simultaneously over a period of 20 seconds during quiet breathing, exercise, and recovery is shown in fig 2 . The linear regression analysis yielded the following equation: $\mathrm{VT}_{\mathrm{OEP}}=1.20 \mathrm{VT}_{\mathrm{SP}}-$ $0.18\left(r^{2}=0.97, \mathrm{p}<0.001\right)$. The mean percentage difference between $\mathrm{VT}_{\mathrm{OEP}}$ and $\mathrm{VT}_{\mathrm{SP}}$ was $-2.8(1.2) \%$ or 31 (14) $\mathrm{ml}$. The difference between the two systems at maximum workload (100\%peak) was $8.4(4.5) \%$ or 93 (17) ml, with the VT values being larger.

Changes in IC from quiet breathing measured by the spirometer $\left(\Delta \mathrm{IC}_{\mathrm{SP}}\right)$ were in good relationship with the EEVcW calculated by the OEP $\left(\Delta \mathrm{EEVCW}_{\mathrm{OEP}}\right)$ during exercise and recovery (fig 3A). Linear regression analysis provided the following equation: $\Delta \mathrm{EECW}_{\mathrm{OEP}}=0.82 \Delta \mathrm{IC}_{\mathrm{SP}}+0.03$ $\left(r^{2}=0.91, \mathrm{p}<0.001\right)$. The mean percentage difference between $\Delta \mathrm{EECW}_{\mathrm{OEP}}$ and $\Delta \mathrm{IC}_{\mathrm{SP}}$ throughout all stages was $7.0(5.8) \%$ or $35(24) \mathrm{ml}$.

In addition, a close correlation was found between $\mathrm{IC}_{\mathrm{OEP}}$ and $\mathrm{IC}_{\mathrm{SP}}$ during all stages (fig $3 \mathrm{~B}$ ). Linear regression analysis provided the following equation: $\mathrm{IC}_{\mathrm{OEP}}=0.65 \mathrm{IC}_{\mathrm{SP}}+0.52$ $\left(r^{2}=0.89, \mathrm{p}<0.001\right)$. The mean percentage difference throughout all stages between $\mathrm{IC}_{\mathrm{OEP}}$ and $\mathrm{IC}_{\mathrm{SP}}$ was 3.8 (1.8)\% or $73(32) \mathrm{ml}$.

\section{Changes in operational Vcw during exercise and recovery}

Two significantly different patterns of change in EEVcw were observed during exercise in our patients (fig 4). Twelve patients exhibited a progressive significant increase in EEVcw during exercise (early hyperinflators, EH) amounting to 750 (90) $\mathrm{ml}$ at Wpeak (fig 4A). In contrast, in all eight remaining patients EEVCW remained unchanged from quiet breathing

Table 2 Mean (SE) peak exercise data of the study population and subgroups

\begin{tabular}{|c|c|c|c|}
\hline & $\begin{array}{l}\text { COPD } \\
(n=20)\end{array}$ & $\begin{array}{l}\text { Early hyperinflators } \\
(n=12)\end{array}$ & $\begin{array}{l}\text { Late hyperinflators } \\
(\mathrm{n}=8)\end{array}$ \\
\hline Wpeak (Watt) & $45(4)$ & $44(7)$ & $47(5)$ \\
\hline Wpeak (\% pred) & 37 (3) & $34(4)$ & $40(5)$ \\
\hline Exercise tolerance (min) & $6.7(0.4)$ & $6.4(0.5)$ & $7.2(0.6)$ \\
\hline$\dot{\mathrm{V}}_{2}(\mathrm{l} / \mathrm{min})$ & $0.83(0.06)$ & $0.81(0.09)$ & $0.84(0.08)$ \\
\hline $\mathrm{V}_{2}(\%$ pred $)$ & $50(4)$ & $47(5)$ & $53(7)$ \\
\hline RER & $1.06(0.05)$ & $1.08(0.02)$ & $1.07(0.07)$ \\
\hline$f_{c}$ (beats $/ \mathrm{min}$ ) & $115(2)$ & $114(4)$ & $116(5)$ \\
\hline$f_{c}(\%$ pred $)$ & $73(2)$ & $72(2)$ & $75(5)$ \\
\hline $\mathrm{SpO}_{2}(\%)$ & $92(1)$ & $93(2)$ & 91 (1) \\
\hline VE $(1 / \mathrm{min})$ & $30.8(2.0)$ & $31.1(2.6)$ & $30.1(2.7)$ \\
\hline VT (I) & $1.24(0.08)$ & $1.22(0.10)$ & $1.28(0.12)$ \\
\hline $\mathrm{fb}$ (breaths/min) & $26(2)$ & $26(3)$ & $25(2)$ \\
\hline Dyspnoea (Borg) & $4.1(0.3)$ & $4.1(0.5)$ & $3.6(0.3)$ \\
\hline Leg fatigue (Borg) & $4.1(0.3)$ & $4.3(0.3)$ & $3.4(0.7)$ \\
\hline$\Delta \mathrm{EIVW}_{\mathrm{V}}$ (I) & $0.97(0.10)$ & $1.17(0.17)$ & $0.66(0.08)^{*}$ \\
\hline$\Delta \mathrm{EEV}_{\mathrm{cw}}$ (l) & $0.53(0.09)$ & $0.75(0.09)$ & $0.21(0.08)^{*}$ \\
\hline VTOEP $/$ IC OEP $(\%)$ & $86(2)$ & $88(2)$ & $83(6)$ \\
\hline IRVcw (I) & $0.19(0.04)$ & $0.14(0.05)$ & $0.26(0.08)$ \\
\hline IRVcw/TLCV & $7(1)$ & $7(1)$ & $7(3)$ \\
\hline
\end{tabular}

Wpeak, peak workload; $\dot{V}_{2}$, oxygen uptake; RER, respiratory exchange ratio; $f_{c}$, cardiac frequency; $\dot{V}_{E}$, minute ventilation; $\mathrm{fb}$, breathing frequency; EIV Cw, end inspiratory chest wall volume; $E E V_{C w}$, end expiratory chest wall volume; IC, inspiratory capacity; OEP, optoelectronic plethysmography; $I R V_{c w}$, inspiratory reserve chest wall

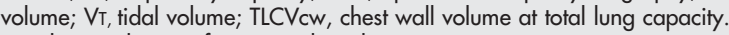
$\Delta$ indicates changes from quiet breathing. *Significant differences between groups. 


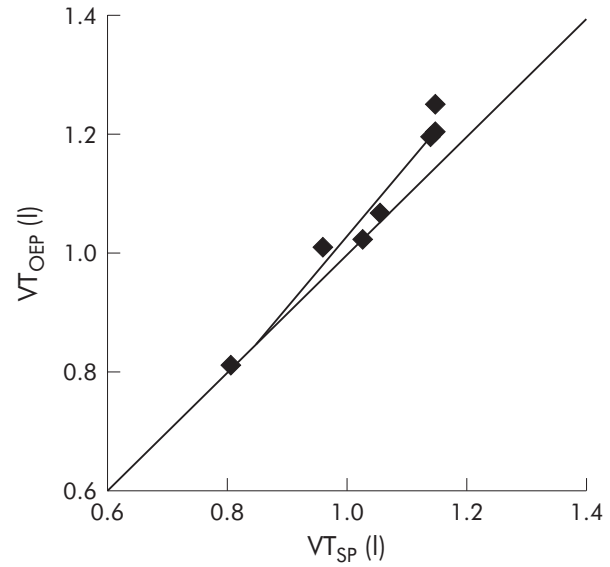

Figure 2 Regression line between tidal volume (VT) from the spirometer ( $\mathrm{VT}_{\mathrm{SP}}$ ) and optoelectronic plethysmography (VTOEP) during quiet breathing, exercise, and recovery. Each point represents the mean value of all 20 patients. Line of identity is also shown.

up to $66 \%$ Wpeak (fig 4B). In the latter group, however, EEVCW was significantly increased by 210 (80) ml compared with quiet breathing at Wpeak (late hyperinflators, LH). The two groups also differed in terms of the recovery pattern: in the EH group EEVcw was still 254 (130) ml higher at 3 minutes into the recovery period than at quiet breathing while, in the LH group, the EEVcw had returned to the level at quiet breathing within 3 minutes of the recovery (fig 4).

In the EH group TLCVCw increased, albeit not significantly, compared with quiet breathing from 66\% Wpeak onwards (fig 4). At Wpeak the increase in TLCVCW from quiet breathing amounted to 198 (95) $\mathrm{ml}$, corresponding to an increase of $0.8(0.1) \%$ of TLCVCw measured at quiet breathing. At 1 minute of recovery TLCVCW was still higher than during quiet breathing (by 153 (64) ml), whereas by 3 minutes of recovery TLCVCW had reached values very close to quiet breathing (fig 4). Similarly, in the LH group, TLCVCW increased by $72(25) \mathrm{ml}$ at Wpeak $(0.3(0.1) \%$ of TLCVCw at quiet breathing) but it did not differ significantly from that recorded during quiet breathing (fig 4).

The pattern of change in EIVcw during exercise did not differ between the groups. EIVCW increased significantly throughout exercise and remained significantly higher than quiet breathing during recovery (fig 4). At Wpeak patients in both groups breathed with a tidal EIVcw that closely approached TLCVCW (table 2), thus restricting further expansion of $\mathrm{VT}_{\mathrm{OEP}}$. During exercise, $\dot{\mathrm{V}}_{\mathrm{E}}$ and $\mathrm{VT}_{\mathrm{OEP}}$ in the $\mathrm{EH}$ group tended to be higher than in the LH group. Nevertheless, volume constraints on $\mathrm{VT}_{\mathrm{OEP}}$ expansion $\left(\mathrm{VT}_{\mathrm{OEP}} / \mathrm{IC}_{\mathrm{OEP}}\right.$, IRVCW/TLCVCW) were similar between the groups, whereas IRVcw reached the same level in both groups (table 2). Symptoms of dyspnoea and leg discomfort did not differ between the groups. Neither resting lung volumes, peak exercise workload, nor gas exchange were significantly different between the two groups (tables 1 and 2).

\section{Compartmental tidal volumes}

The volume variations for the abdominal compartment were significantly different between groups during exercise and recovery (fig 4 middle panels). In the EH group the increase in EEVcw with increasing work rate was almost entirely attributable to the significant increase in end expiratory Vrc with no significant contribution from Vab (fig 4, top left and middle panels). In contrast, in the LH group there was no significant change in EEVCW up to $66 \%$ Wpeak; this was
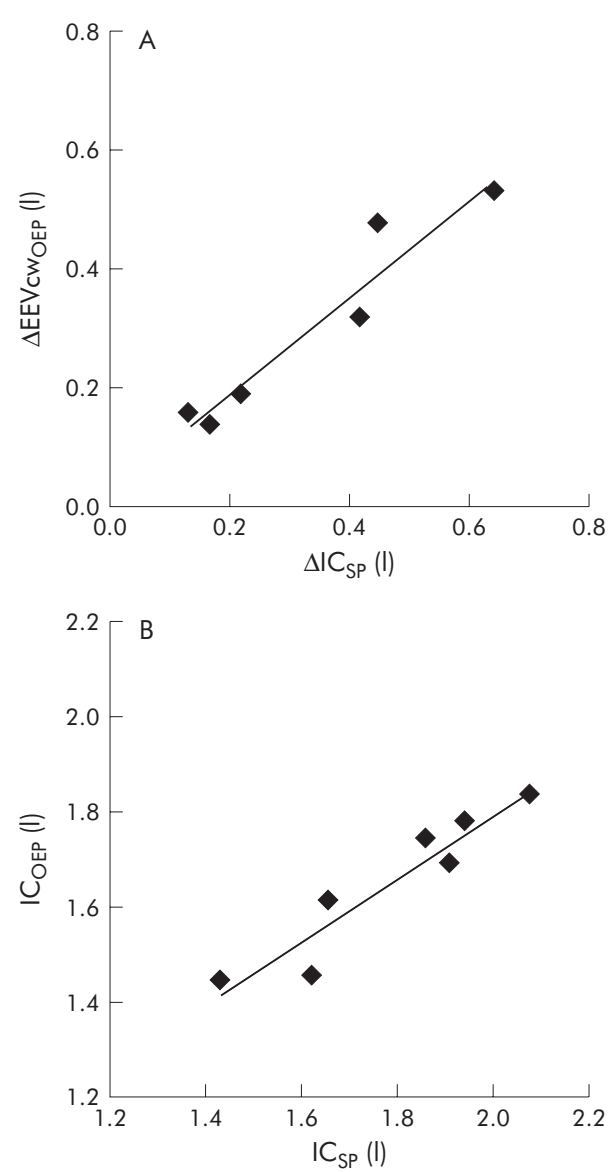

Figure 3 (A) Regression line between volume changes from quiet breathing in inspiratory capacity measured by the spirometer $\left(\Delta \mid C_{S P}\right)$ and end expiratory chest volume calculated by optoelectronic plethysmography $\left(\triangle \mathrm{EEV} \mathrm{Cw}_{\mathrm{OEP}}\right)$ throughout all stages. Each point represents the mean value of all 20 patients. (B) Regression line between the inspiratory capacity measured by the spirometer $\left(I_{S P}\right)$ and the inspiratory capacity calculated by the optoelectronic plethysmography (ICOEP) at quiet breathing, during exercise and recovery. Each point represents the mean value of all 20 patients.

attributed to the significant decrease seen in end expiratory Vab during exercise (fig 4, bottom middle panels). In the EH group $\mathrm{VT}_{\mathrm{OEP}}$ expansion was due to a progressive increase in end inspiratory Vrc and Vab whereas in the LH group the increase in $\mathrm{VT}_{\mathrm{OEP}}$ was achieved by an increase in end inspiratory Vrc and a decrease in Vab (fig 4).

In both groups, 3 minutes into recovery $\mathrm{VT}_{\mathrm{OEP}}$ remained significantly higher than at quiet breathing, mainly as a result of increased end inspiratory Vrc in both groups. In contrast, within 3 minutes of recovery the end expiratory Vrc in both the EH and LH groups was not significantly different than at quiet breathing.

Throughout incremental exercise sensations of dyspnoea and leg discomfort tended to be higher in the EH group than in the LH group (fig 5). However, the differences between the groups were not significant.

\section{DISCUSSION}

The main findings of this study are:

- In patients with severe COPD there are two distinct patterns of change in the chest wall volume response to exercise: in the EH group EEVcw progressively increases throughout exercise while in the LH group it remains 

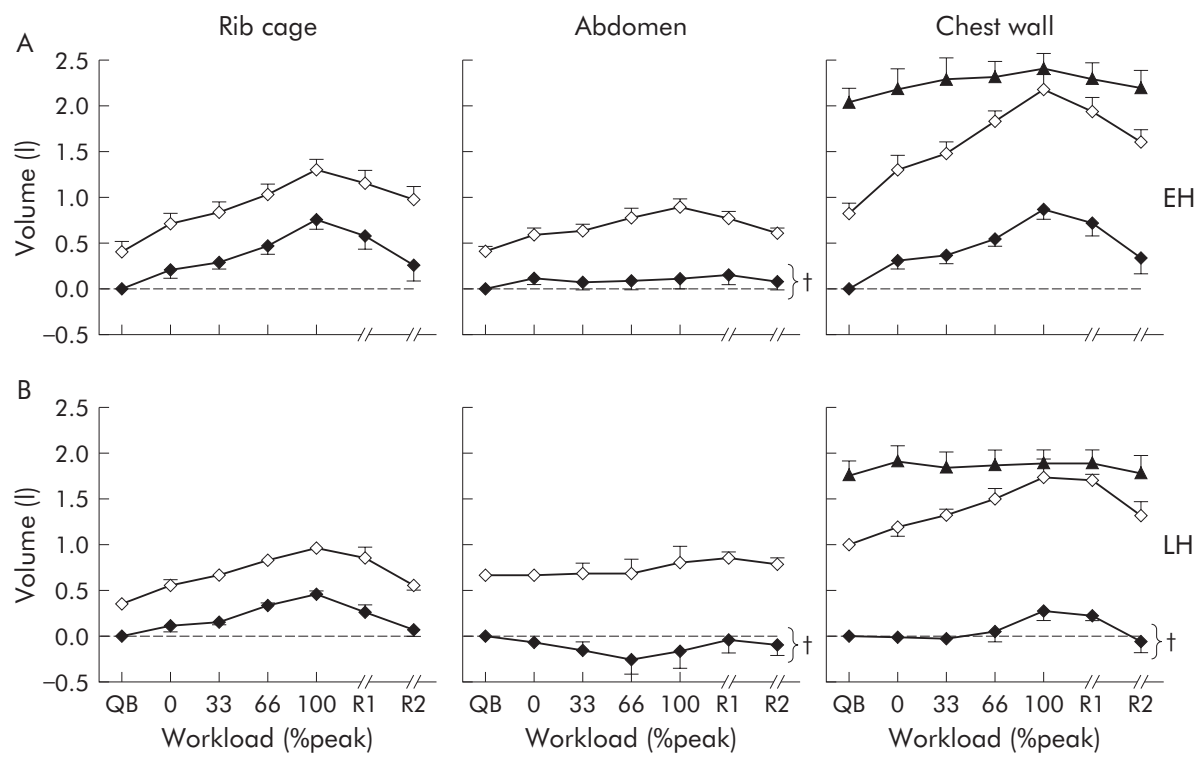

Figure 4 Volumes of the rib cage and the abdominal compartments and of the total chest wall in (A) early hyperinflators (EH) and (B) late hyperinflators (LH) expressed in absolute values during quiet breathing (QB), exercise, and recovery (R1 and R2). Open circles indicate end of inspiration; closed circles indicate end of expiration; triangles indicate chest wall volumes at total lung capacity. †Significant differences in time points between groups.

unchanged up to $66 \%$ Wpeak, but increases significantly at Wpeak.

- Although at the limit of tolerance the increase in EEVCW was significantly greater in the EH than in the LH group, both reached similar values of Wpeak, IRVCW and dyspnoea.

- Groups did not differ in terms of resting lung volumes or exercise tolerance measures.
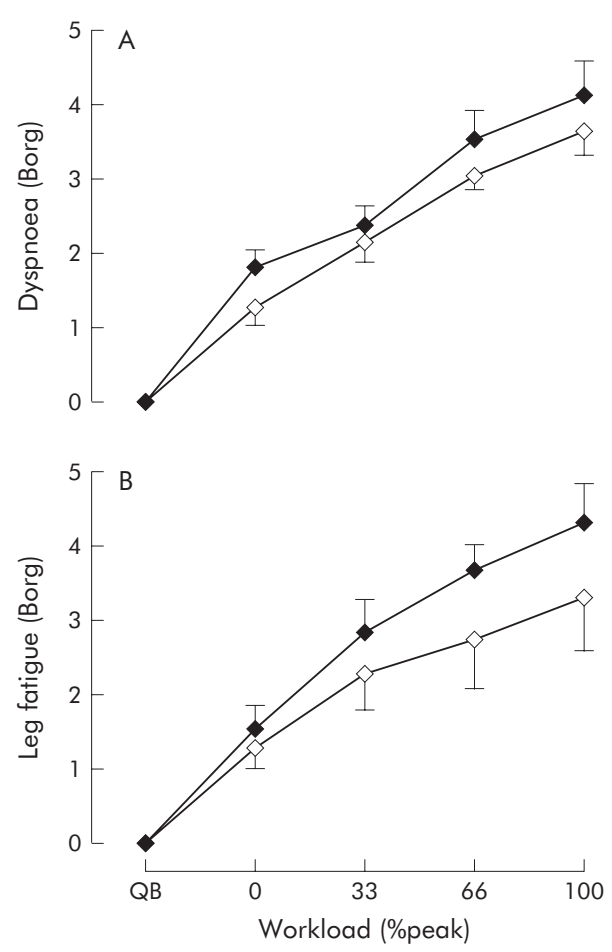

Figure 5 Perceptions of (A) dyspnoea and (B) leg discomfort at quiet breathing (QB) and during exercise between early (closed symbols) and late hyperinflators (open symbols).
- After exercise the EEVcw did not return to the pre-exercise value by 3 minutes in the EH group.

In healthy subjects, in whom expiratory flow limitation (EFL) is absent, EELV decreases progressively during exercise..$^{13}$ In contrast, the progressive increase in EELV that is typically observed in patients with severe COPD during exercise is mainly dictated by EFL. ${ }^{12}{ }^{18}$ Koulouris and colleagues ${ }^{12}$ have shown that if EFL is present during resting breathing, any further increase in ventilation during exercise is associated with progressive dynamic hyperinflation. The progressive increase in EEVcw observed in the EH group ( 12 patients) with increasing exercise level presumably reflects the presence of EFL already at rest. On the other hand, eight patients exhibited hyperinflation only at Wpeak. This suggests that in these subjects EFL started only after $66 \%$ Wpeak, in line with previous findings ${ }^{12}$ which indicated that some COPD patients do not reach EFL up to two thirds of Wpeak. Furthermore, it should be noted that in our LH patients, the end expiratory Vab decreased progressively in the range of 0-66\% Wpeak. The decreased end expiratory Vab during exercise reflected increased abdominal muscle activity. It has been postulated that such a contraction is beneficial because of lengthening of the diaphragm resulting in improved generation of a negative pleural pressure (better position of the length-tension relationship). ${ }^{19}{ }^{20}$ Although, end expiratory Vab was reduced over this exercise range, EEVcw did not change as there was a simultaneous increase in end expiratory Vrc.

Aliverti et $a l^{11}$ have also reported that not all patients with COPD hyperinflate during exercise. They studied 20 patients during incremental exercise: 12 were $\mathrm{EH}$ similar to the present study, while in eight the EEVcw actually decreased from early exercise. These subjects were, however, different from the LH subjects of the present study and those of Koulouris et al. ${ }^{12}$ Their $\mathrm{FEV}_{1}$ averaged $50 \%$ of predicted compared with our value of $37 \%$, and their exercise performance was very poor with a Wpeak of only $20 \mathrm{~W}$ compared with $40 \mathrm{~W}$ in the LH group. In the present study the overall group of patients is comparable, at least in terms of $\mathrm{FEV}_{1}$, to that reported by Aliverti et $a l^{11}$ for the 
hyperinflators (having an $\mathrm{FEV}_{1}$ of $39 \%$ predicted). This is probably the reason why we were not able to identify any "euvolumic" patients as described by Aliverti et al. ${ }^{11}$

Furthermore, there were important differences in chest wall kinematics in their non-hyperinflators ${ }^{11}$ compared with our LH group. They found no increase in EIVcw as exercise workload increased and hence the increase in $\mathrm{VT}_{\mathrm{OEP}}$ during exercise was solely due to the decrease in EEVcw, presumably reflecting absence of EFL throughout exercise. In addition, at Wpeak there was a large IRVCW amounting to approximately $1.3 \mathrm{l}$, while in our subjects it was only $0.26 \mathrm{l}$. Neither Koulouris et $a l^{12}$ nor O'Donnell et al found patients with such a high inspiratory reserve volume at the limit of tolerance as Aliverti et al. ${ }^{11}$ In our study $\mathrm{VT}_{\mathrm{OEP}}$ increased with exercise entirely by an increase in EIVcw so that, at Wpeak, EIVCW was very close to TLCVCW and IRVCW was minimal. We found no decrease in EEVcw and the decrease in end expiratory Vab was considerably less than in their patients. It is therefore possible that, besides the different degree of EFL experienced by patients in the two studies, expiratory muscle recruitment was more in their patients than in ours, leading to a greater work of breathing.

Accordingly, when the findings of the present study are compared with those of Aliverti et al, ${ }^{11}$ it can be suggested that, during the natural history of COPD, patients pass through a stage with moderate impairment of expiratory flow rates so that exercise does not impose dynamic hyperinflation. With further disease progression, manifested by decreasing $\mathrm{FEV}_{1}$, dynamic hyperinflation might be accompanied by lesser degrees of expiratory muscle recruitment and increased dynamic hyperinflation. Longitudinal studies will be required to determine if this hypothesis is correct.

The present study provides, for the first time, simultaneous changes in Vcw at the end of inspiration, expiration, and at TLC during symptom limited exercise in patients with severe COPD. Interestingly, at Wpeak tidal EIVCW closely approached TLCVCW in both EH and LH. Accordingly, exercise limitation was associated with the fixed mechanical constraint set by the reduced IRVCW rather than the magnitude of the change in EEVcw per se. The increase in dynamic hyperinflation during exercise is therefore not the only mechanism limiting exercise capacity in patients with severe COPD.

We also observed that TLCVCw in both $\mathrm{EH}$ and $\mathrm{LH}$ increased at Wpeak from quiet breathing, albeit not significantly (EH: by 198 (95) $\mathrm{ml}$ or $0.8(0.1) \%$ of TLCVCW measured during quiet breathing; LH: by 72 (25) $\mathrm{ml}$ or 0.3 $(0.1) \%$ of TLCVCw measured during quiet breathing). The magnitude of these changes in TLCVCw in both groups during exercise is in agreement with previous suggestions that small changes in TLC may occur during exercise because hyperinflation can cause an increase in lung distensibility. ${ }^{21-23}$ Accordingly, changes in TLCVCW tended to be larger in EH, possibly because they were more hyperinflated than the LH. It should be noted, however, that changes in chest wall volumes include changes in gas volume, gas compression, and blood volume. ${ }^{13}$ The progressive increase in TLCVCW seen in both groups could therefore be the result of all of these factors, which may in turn explain, at least in part, the small discrepancies found between the changes in volumes recorded at the mouth by the spirometer and those calculated from the chest wall signals (figs 2 and 3 ).

In the EH group EEVCW was increased by 254 (130) ml above baseline 3 minutes after exercise. This is in agreement with that recently reported by O'Donnell et $a^{24}$ who found that IC 3 minutes into recovery from symptom limited exercise was greater by 250 (35) $\mathrm{ml}$ than at baseline. The present study extends these findings by showing that, in the EH group, the greater degree of dynamic hyperinflation and air trapping during exercise should have enhanced the threshold loading mainly of the muscles of the rib cage compartment so that during recovery the function of these muscles would take longer to return to baseline. Furthermore, the delayed recovery of dynamic hyperinflation has important clinical implications when designing rehabilitative exercise training regimes for patients with severe COPD, especially if high intensity interval exercise is chosen..$^{25} 26$

In conclusion, we found that patients with severe COPD fall into two groups: those who hyperinflate early in exercise and those who hyperinflate late. Despite this different pattern, exercise capacity is similar, probably reflecting the fact that both groups closely approached their TLC at Wpeak.

\section{Authors' affiliations}

I Vogiatzis, O Georgiadou, S Golemati, E Kosmas, E Kastanakis, A Koutsoukou, S Nanas, S Zakynthinos, Ch Roussos, National and Kapodistrian University of Athens, Department of Critical Care Medicine and Pulmonary Services, Evangelismos Hospital, "M Simou and G P Livanos Laboratories", Athens, Greece

I Vogiatzis, O Georgiadou, N Geladas, Department of Physical Education and Sport Science, Athens, Greece

A Aliverti, Dipartimento di Bioingegneria, Politecnico di Milano, Milano, Italy

This work was supported by the European Community CARED FP5 project (contract n. QLG5-CT-2002-0893) and by the Thorax Foundation.

Competing interests: none declared.

\section{REFERENCES}

1 O'Donnell DE, Webb KA. Exertional breathlessness in patients with chronic airflow limitation: the role of lung hyperinflation. Am J Respir Crit Care Med 1993;148:1351-7.

2 Belman MJ, Botnick WC, Shin JW. Inhaled bronchodilators reduce dynamic hyperinflation during exercise in patients with chronic obstructive pulmonary disease. Am J Respir Crit Care Med 1996;153:967-75.

3 O'Donnell DE, Bertley JC, Webb KA, et al. Mechanism of relief of exertional breathlessness following unilateral bullectomy and lung volume reduction surgery in advanced chronic airflow limitation. Chest 1996; 110:18-27.

4 Dolmage TE, Waddell TK, Maltais F, et al. The influence of lung volume reduction surgery on exercise in patients with COPD. Eur Respir $J$ 2004:23:275-80.

5 O'Donnell DE, Lam M, Webb KA. Measurement of symptoms, lung hyperinflation and endurance during exercise in chronic obstructive pulmonary disease. Am J Respir Crit Care Med 1998;158:1557-65.

6 O'Donnell DE, Revill SM, Webb KA. Dynamic hyperinflation and exercise intolerance in chronic obstructive pulmonary disease. Am J Respir Crit Care Med 2001; 164:770-7.

7 Stubbing DG, Pengelly LD, Morse JLC, et al. Pulmonary mechanics during exercise in subjects with chronic airflow obstruction. J Appl Physiol 1980;49:511-5.

8 Yan S, Kaminski D, Sliwinsky P. Reliability of inspiratory capacity for estimating end-expiratory lung volume changes during exercise in patients with chronic obstructive pulmonary disease. Am J Respir Crit Care Med 1997; 156:55-9.

9 Potter WA, Olafsson S, Hyatt RE. Ventilatory mechanics and expiratory flow limitation during exercise in patients with obstructive lung disease. J Clin Invest 1971:50:910-9.

10 Dodd DS, Brancatisano T, Engel LA. Chest wall mechanics during exercise in patients with severe chronic airflow obstruction. Am Rev Respir Dis 1984;129:33-8.

11 Aliverti A, Stevenson N, Dellaca RL, et al. Regional chest wall volumes during exercise in chronic obstructive pulmonary disease. Thorax 2004;59:210-6.

12 Koulouris NG, Dimopoulou I, Valta P, et al. Detection of expiratory flow limitation during exercise in COPD patients. J Appl Physiol 1997;82:723-31.

13 Iandelli I, Aliverti A, Kayser B, et al. Determinant of exercise performance in normal men with externally imposed expiratory flow limitation. J Appl Physiol 2002;92:1943-52.

14 Aliverti A, Cala SJ, Duranti R, et al. Human respiratory muscle actions and control during exercise. J Appl Physiol 1997;83:1256-69.

15 Vogiatzis I, Aliverti A, Golemati S, et al. Respiratory kinematics by optoelectronic plethysmography during exercise in healthy subjects. Eur J Appl Physiol 2005;93:581-7.

16 Gardner RM, Hankinson JL, Clausen JL, et al. American Thoracic Society standardization of spirometry - 1987 update. Am Rev Respir Dis 1987; 136:1285-98.

17 Borg GAV. Psychophysical bases of perceived exertion. Med Sci Sports Exerc 1982;14:377-81. 
18 Calverley PMA, Koulouris NG. Flow limitation and dynamic hyperinflation: key concepts in modern respiratory physiology. Eur Respir $J$ 2004;25:186-99.

19 Grimby G, Goldman M, Mead J. Respiratory muscle action inferred from rib cage and abdominal VP partitioning. J Appl Physiol 1976;41:739-51.

20 Goldman M, Grimby G, Mead J. Respiratory muscle action inferred from rib cage and abdominal VP partitioning. J Appl Physiol 1976;41:752-63.

21 Hanson JS, Tabakin BS, Caldwell EJ. Response of lung volumes and ventilation to posture change and upright exercise. J Appl Physiol 1962;17:783-6.

22 Hillman DR, Finucane KE. The effect of hyperinflation on lung elasticity in healthy subjects. Respir Physiol 1983;54:295-305.
23 Glaister DH, Schroter RC, Sudlow MF, et al. Bulk elastic properties of excised lungs and the effect of a transpulmonary pressure gradient. Respir Physiol 1973; 17:347-64.

24 O'Donnell DE, Johnson B, Richter K, et al. Inspiratory capacity and dyspnoea during recovery from symptom-limited exercise in COPD patients treated with tiotropium. Eur Respir J 2004;24(Suppl 48):214s.

25 Vogiatzis I, Nanas S, Roussos C. Interval training as an alternative modality to continuous exercise in patients with COPD. Eur Respir J 2002;20:12-9.

26 Vogiatzis I, Nanas S, Kastanakis E, et al. Dynamic hyperinflation and tolerance to interval exercise in patients with advanced COPD. Eur Respir J 2004; $24: 358-63$

\section{LUNG ALERT}

Is adjuvant chemotherapy for non-small cell lung cancer here to stay?

$\Delta$ Winton T, Livingston R, Johnson D, et al. Vinorelbine plus cisplatin vs observation in resected non-small cell lung cancer. N Engl J Med 2005;352:2589-97

$\mathrm{n}$ this study patients with completely resected stage IB or stage II non-small cell lung cancer were randomised to either adjuvant chemotherapy with vinorelbine and cisplatin $(n=242)$ or to observation $(n=240)$. The primary end point was overall median survival which was significantly prolonged in the chemotherapy group (94 $v 73$ months; adjusted $\mathrm{p}=0.04)$. This corresponds to an overall survival advantage at 5 years of 15 percentage points $(p=0.03)$. Fewer patients in the chemotherapy group had disease recurrence $(36.0 \%$ $v 49.6 \%, \mathrm{p}=0.003)$. While subgroup analysis of stage IB patients did not show a significant improvement in survival, the overall analysis showed disease stage not to be a significant predictor of treatment effect. Improved survival was associated with chemotherapy and squamous histology, whereas shorter survival was associated with older age, male sex, and pneumonectomy compared with lesser resection. Side effects from chemotherapy were seen in many patients, but in comparable numbers to other reports: there were two deaths $(0.8 \%)$ and febrile neutropenia was documented in $7 \%$.

This study has continued the recent trend of showing survival advantage with adjuvant chemotherapy and demonstrates a greater benefit than previous reports. This may be due to the sole use of a modern chemotherapy regimen compared with previous studies. Is adjuvant chemotherapy now to be considered the standard of care for such patients undergoing complete resection? Probably, although further work should be done to delineate which patients are likely to obtain the greatest benefits while hopefully avoiding the severe morbidity which can be associated with chemotherapy.

T J Warke

Consultant Respiratory Physician, Royal Victoria Hospital, Belfast, UK; tim@dsl.pipex.com 